\title{
Immunohistochemical analysis of undifferentiated and poorly-differentiated head and neck malignancies at a tertiary hospital in Nigeria
}

\author{
Akinyele O Adisa ${ }^{1 *}$, Abideen O Oluwasola², Bukola F Adeyemi', Bamidele Kolude 1 , Effiong EU Akang², \\ Jonathan O Lawoyin ${ }^{1}$
}

\begin{abstract}
Absract
This is a retrospective analysis of poorly-differentiated head and neck malignancies at University College Hospital, Ibadan.

Eighty-six poorly-differentiated neoplasms were categorized as carcinomas, sarcomas, lymphomas or neuroendocrine cancers with a panel of 7 antibodies (cytokeratin AE1/AE3, vimentin, desmin, myogenin, leukocyte common antigen and neuron-specific enolase). Immunohistochemical and original hematoxylin-eosin diagnoses were contrasted.

The male: female ratio was 2.5:1, with mean age of 38.9 years. Nasopharynx, nose and maxillofacial bones were the most common locations. Immunohistochemistry confirmed $54.8 \%$ of carcinomas, $70.6 \%$ of sarcomas and $80 \%$ of lymphomas.

Hematoxylin-eosin was able to distinguish between sarcoma and lymphoma but differentiation between a carcinoma and neuroendocrine lesion was poor. Further studies are required to maximize the role of immunohistochemistry as an ancillary diagnostic tool in the West African sub-region.
\end{abstract}

\section{Introduction}

Histological examination plays a central role in diagnosis, classification, grading and staging of malignancy. Difficulties arise from the subjective nature of histological analysis that are influenced by the practitioner's experience, bias and training. With poorly-differentiated neoplasms, inter- and intra-observer variability can be high [1]. Immunohistochemistry has greatly assisted in the identification of tumors that cannot be accurately identified using routine histopathological procedures [2]. In one study of more than 100 anaplastic tumors, the hematoxylin-eosin diagnosis of carcinoma or lymphoma was revised in approximately $50 \%$ of cases following immunohistochemical analysis [3].

In some undifferentiated tumors, subtle features of epithelial versus mesenchymal differentiation can often be appreciated, which assist the immunohistochemical

\footnotetext{
* Correspondence: perakin80@hotmail.com

'Department of Oral Pathology, University College Hospital, Ibadan, Oyo State, Nigeria

Full list of author information is available at the end of the article
}

approach to these tumors. Some tumors, however, may not fit into either of these two categories because of their overlapping histological features [4]. Nevertheless, making the correct histopathological diagnosis is essential in deciding the appropriate therapy $[5,6]$.

The immunohistochemical evaluation of undifferentiated tumors should first aim at a broad lineage determination of the neoplasia. Based on the result of the screening panel, a more detailed or specific panel should then be applied to further sub classify the tumor or to confirm a particular diagnosis [4]. The thrust of this study is to evaluate the accuracy of histopathological diagnosis in the broad lineage determination of undifferentiated/ poorly-differentiated neoplasms of the head and neck.

\section{Methodology}

1192 head and neck malignancies (oral and nasal cavities, paranasal sinuses, oropharynx, nasopharynx, hypopharynx, larynx, trachea, ear and salivary glands) were retrieved from the archives of the Pathology and Oral Pathology departments of the University College
C Biomed Central

C 2010 Adisa et al; licensee BioMed Central Ltd. This is an Open Access article distributed under the terms of the Creative Commons Attribution License (http://creativecommons.org/licenses/by/2.0), which permits unrestricted use, distribution, and reproduction in any medium, provided the original work is properly cited. 
Hospital, Ibadan, Nigeria between 1990 and 2008. 142 poorly-differentiated and undifferentiated neoplasms including anaplastic (undifferentiated) or poorly-differentiated carcinomas, anaplastic large cell lymphomas, pleomorphic sarcomas, malignant fibrous histiocytoma, esthesioneuroblastoma and spindle cell sarcomas were selected. Cases where the original paraffin block could not be obtained were excluded from analysis. Only 86 of the 142 undifferentiated and poorly-differentiated head and neck malignancies diagnosed during the study period satisfied the inclusion criteria.

Freshly prepared sections from each case were stained with hematoxylin-eosin ( $\mathrm{H} \& \mathrm{E})$ and a panel of antibodies to leukocyte common antigen (CD45), cytokeratin AE1/ AE3, vimentin, desmin, myogenin and neuron-specific enolase (NSE) using the specifications of the manufacturer (Dako Cytomation, USA).

The sections for immunohistochemistry were de-paraffinized, hydrated and then rinsed in Phosphate Buffered Solution (PBS). They were immersed in heat induced epitope retrieval citrate buffer diluted to 1:10 with distilled water and incubated at $90^{\circ} \mathrm{C}$ for 1 hour. They were then placed in fresh citrate, cooled in water for 20 minutes and then rinsed in PBS. Positive controls (skin for cytokeratin AE1 or AE3, tonsils for CD45; neural tissue for Neuron-specific enolase; skeletal muscle for Myogenin and Vimentin; and smooth muscle for desmin) and negative controls were employed for each antibody. 3\% hydrogen peroxide was added to each section for 10 minutes and the sections were rinsed in $0.1 \%$ PBS. The specimens were incubated for an hour with 40-130 $\mu \mathrm{l}$ of appropriately diluted Dako mouse primary antibody, followed by incubation with undiluted labeled polymer Horse Radish Peroxidase conjugated antimouse secondary antibody for 30 minutes. One $\mathrm{ml}$ of Diaminobenzidene solution was added to cover the specimen, followed by incubation in a humidity chamber for 15 minutes. The sections were then immersed in aqueous hematoxylin and rinsed in distilled water. The tissue was then dehydrated and subsequently rinsed with xylene. DPX (Distyrene, Plasticizer and Xylene) mounting fluid was then applied and a cover slip placed.

All the seven antibodies used in the panel for one specimen were reviewed sequentially and the pattern and intensity of staining was observed and scored as: negative $(0)$, weakly positive $(+1)$, moderately positive $(+2)$ and strongly positive $(+3)$ [7]. The slides were reviewed without reference to initial histology diagnosis to eliminate bias. The final immunohistochemical findings were then correlated with the H\&E stained slides in order to arrive at a final diagnosis.

The data was analyzed using version 16 of the Statistical Package for Social Sciences (SPSS16). Qualitative data were compared using chi-square statistics.
Quantitative data were summarized using mean, standard deviation and confidence interval and compared using student $\mathrm{t}$ - and/or one-way analysis of variance test. The level of significance was set at $p<0.05$. Sensitivity and specificity were calculated using immunohistochemistry as the gold standard to which the original $\mathrm{H} \& \mathrm{E}$ diagnosis was compared. The positive predictive value, negative predictive value, accuracy and degree of agreement were also determined. For degree of agreement, Kappa value $>0.75=$ excellent agreement, $0.4-0.75=$ fair to good agreement and $<0.4=$ moderate to poor agreement [8].

\section{Results}

The cases comprised 62 (72.1\%) males and 24 (27.9\%) females. The mean age was $38.9(\mathrm{SD} \pm 15.9)$ with peak occurrence between 25-44 years. The nasopharynx (47.7\%), nose (12.8\%) and maxillofacial bones $(12.8 \%)$ were the most common locations.

The original hematoxylin-eosin diagnoses are shown in figure 1 . These diagnoses were confirmed by immunohistochemistry in $34(54.8 \%)$ of the 62 carcinomas, 12 (70.6\%) of the 17 sarcomas, four ( $80 \%)$ of the 5 lymphomas and one of the 2 neuroendocrine malignancies (Table 1 ).

Table 2 summarizes the clinical profile of the 33 cases in which there was discordance between the original $\mathrm{H} \& \mathrm{E}$ diagnosis and final diagnosis. Some of these discordant diagnoses are also depicted in figures $2,3,4,5$, 6 and 7. Sarcomas, neuroendocrine carcinomas and lymphomas were most often misdiagnosed as carcinomas. There was no obvious correlation between age, gender or site distribution, as compared to diagnosis.

The sensitivity of histology was highest for carcinomas (97.1\%) and least for neuroendocrine lesions (14.2\%). Specificity of histology was highest for neuroendocrine lesions (98.6\%) and lymphomas (98.4\%) and least for carcinomas $(47.7 \%)$. The positive predictive values of histology was highest for sarcomas and lymphomas (80\% each), while the negative predictive value was highest for carcinomas (95.4\%). Accuracy of histology was highest for the neuroendocrine tumors (91.1\%). The level of agreement between histology and immunohistochemistry, given by the kappa values, was highest for sarcomas (54\%) (Table 3).

\section{Discussion}

In the present study, the original histological diagnosis of 62 lesions was carcinoma, but only 34 (54.8\%) were confirmed by immunohistochemistry. This proportion is higher than the $27.9 \%$ confirmation rate of carcinomas in a study by Bianchini et al in Italy [9]. Lesions that were confused with carcinomas included 8 (12.9\%) sarcomas, 8 (12.9\%) lymphomas, 6 (9.7\%) neuroendocrine tumors and 1 carcinosarcoma. Gatter [10] also reported 


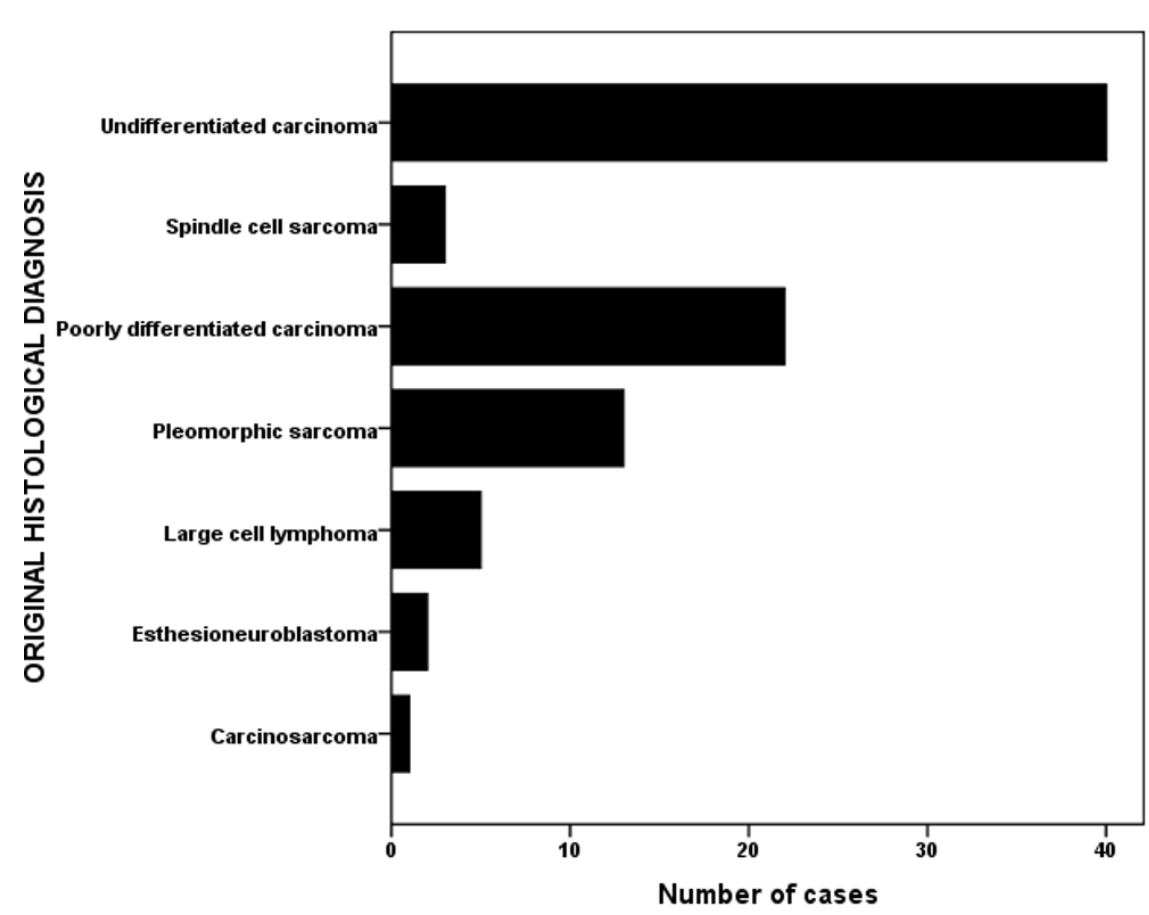

Figure 1 Histological diagnoses of 86 poorly differentiated/undifferentiated head and neck malignancies

in a study that 29 cases (67.4\%) from 43 cases initially thought to be anaplastic carcinomas were revised as lymphomas by immunohistochemistry. This demonstrates the value of immunohistochemistry in distinguishing between anaplastic carcinomas and malignant lymphomas of the head and neck, as the latter is much more amenable to treatment than the former. This study thus corroborates other studies which suggest that immunohistochemical technique has a role in the definition of undifferentiated tumors.

Eighty percent of lymphomas diagnosed by histology in this study were confirmed by immunohistochemistry and this is comparable to the $66 \%$ reported confirmation of lymphomas in another study [3].

After the immunohistochemical analysis, almost 10\% of the lesions thought to be undifferentiated carcinomas were revised to neuroendocrine carcinomas. More than $60 \%$ of these revised lesions were found either in the nose or nasopharynx and occurred more commonly in males (66.7\%). Histopathological differentiation of undifferentiated carcinoma from neuroendocrine carcinoma is challenging and is significantly aided by immunohistochemistry [11].

The present study had inconclusive diagnosis by immunohistochemistry in $8.1 \%$ of cases, Bianchini et al reported $18.6 \%$ and Gatter reported $6.7 \%$ inconclusive results. This could be due to technique differences, different antigen retrieval methods or the absence of the antigen suspected. Use of inappropriate antibodies may also be responsible for absence of immunoreactivity. In addition, some poorly-differentiated tumors might require other techniques such as electron microscopy and molecular studies before an accurate diagnosis can be achieved [12,13].

In this study, histology, to a reasonable extent is able to determine if a sarcoma or lymphoma is present or if

Table 1 Comparison of histology diagnosis with immunohistochemical assessment

\begin{tabular}{llllllll}
\hline & & \multicolumn{5}{c}{ IMMUNOHISTOCHEMISTRY DIAGNOSIS } \\
HISTOLOGY DIAGNOSIS & CA & SARC & LYMPH & NE-CA & CA-SARC & INCONCLUSIVE & TOTAL \\
\hline CA & 34 & 8 & 8 & 6 & 1 & 5 & 62 \\
SARC & 1 & 12 & 2 & 0 & 0 & 2 & 17 \\
LYMPH & 0 & 1 & 4 & 0 & 0 & 0 & 5 \\
NE-CA & 0 & 1 & 0 & 1 & 0 & 0 & 2 \\
TOTAL & 35 & 22 & 14 & 7 & 1 & 7 & 86 \\
\hline
\end{tabular}

KEY: CA- carcinoma, SARC- sarcoma, LYMPH- lymphoma, NE-CA- neuroendocrine carcinoma. 
Table 2 Age group, gender and topography of head and neck cancers in which the final diagnosis was revised after immunohistochemistry

\begin{tabular}{|c|c|c|c|c|}
\hline $\begin{array}{l}\text { ORIGINAL HISTOLOGICAL } \\
\text { DIAGNOSIS }\end{array}$ & $\begin{array}{l}\text { FINAL DIAGNOSIS AFTER } \\
\text { IMMUNOHISTOCHEMISTRY }\end{array}$ & $\begin{array}{l}\text { AGE GROUP } \\
\text { (years) }\end{array}$ & GENDER & TOPOGRAPHY \\
\hline Carcinoma & Neuroendocrine carcinoma & $25-44$ & Male & Lymph node \\
\hline Carcinoma & Neuroendocrine carcinoma & $45-64$ & Female & Nose \\
\hline Carcinoma & Neuroendocrine carcinoma & $45-64$ & Male & Nasopharynx \\
\hline Carcinoma & Neuroendocrine carcinoma & $25-44$ & Female & Nose \\
\hline Carcinoma & Neuroendocrine carcinoma & $15-24$ & Male & Palate \\
\hline Carcinoma & Neuroendocrine carcinoma & $\geq 65$ & Male & Nasopharynx \\
\hline Carcinoma & Sarcoma & $25-44$ & Male & Face/scalp \\
\hline Carcinoma & Sarcoma & $15-24$ & Male & Nasopharynx \\
\hline Carcinoma & Sarcoma & $45-64$ & Male & Lymph node \\
\hline Carcinoma & Sarcoma & $25-44$ & Male & Nasopharynx \\
\hline Carcinoma & Sarcoma & $25-44$ & Male & Nose \\
\hline Carcinoma & Sarcoma & $15-24$ & Male & Nose \\
\hline Carcinoma & Sarcoma & $45-64$ & Male & Nasopharynx \\
\hline Carcinoma & Sarcoma & $45-64$ & Female & $\begin{array}{l}\text { Maxillofacial } \\
\text { bone }\end{array}$ \\
\hline Carcinoma & Lymphoma & $\geq 65$ & Male & Nasopharynx \\
\hline Carcinoma & Lymphoma & $45-64$ & Male & Nasopharynx \\
\hline Carcinoma & Lymphoma & $25-44$ & Male & Oropharynx \\
\hline Carcinoma & Lymphoma & - & Male & Oropharynx \\
\hline Carcinoma & Lymphoma & $15-24$ & Male & $\begin{array}{l}\text { Maxillofacial } \\
\text { bone }\end{array}$ \\
\hline Carcinoma & Lymphoma & $45-64$ & Male & Nose \\
\hline Carcinoma & Lymphoma & $25-44$ & Female & Nasopharynx \\
\hline Carcinoma & Lymphoma & $45-64$ & Female & Nasopharynx \\
\hline Carcinoma & Carcinosarcoma & $45-64$ & Male & Nasopharynx \\
\hline Sarcoma & Carcinoma & $45-64$ & Female & Face/scalp \\
\hline Sarcoma & Lymphoma & $15-24$ & Male & $\begin{array}{l}\text { Maxillofacial } \\
\text { bone }\end{array}$ \\
\hline Sarcoma & Lymphoma & $25-44$ & Male & $\begin{array}{l}\text { Maxillofacial } \\
\text { bone }\end{array}$ \\
\hline Lymphoma & Sarcoma & $25-44$ & Male & Nose \\
\hline Neuroendocrine carcinoma & Sarcoma & $45-64$ & Male & Nasopharynx \\
\hline
\end{tabular}

they are absent. However it cannot, with a good degree of certainty, determine if a carcinoma or a neuroendocrine tumor is present, although it can exclude them fairly accurately. The high number of carcinomas seen in this study therefore suggests an over-diagnosis of carcinomas by histology. For correct management to be instituted for any malignant lesion it must be diagnosed accurately. In this study the diagnostic accuracy of histology for carcinomas is $69.6 \%$. This means that almost $70 \%$ of the time, histology will diagnose carcinomas accurately. The diagnostic accuracy for sarcomas, lymphomas and neuroendocrine tumors is $83.5 \%, 86 \%$ and 91.1\% respectively. Therefore further ancillary tests will be needed to resolve diagnostic doubts.

The level of agreement in this study between morphological classification by histology and immunohistochemical assessment was fair for sarcomas (kappa =
0.54), moderate for carcinomas and lymphomas (kappa $=0.42$ ), and poor for neuroendocrine tumors (kappa $=$ 0.18). However, a larger sample of neuroendocrine tumors will be required before any affirmative deductions can be made about them.

Undifferentiated carcinomas were the most prevalent group in this study constituting $64 \%$ of the undifferentiated malignancies and $7.6 \%$ of all head and neck malignancies. A study by Gatter et al [10] in the United Kingdom recorded poorly-differentiated lymphomas (44.2\%) as the most prevalent undifferentiated tumors. Bianchini et al [9] however reported in their study that the most prevalent cell pattern for poorly-differentiated tumors was the round cell pattern (51\%). They only grouped these lesions into their histogenetic lineage after immunohistochemistry and not before [9]. In practice however a protocol should be developed, guiding 


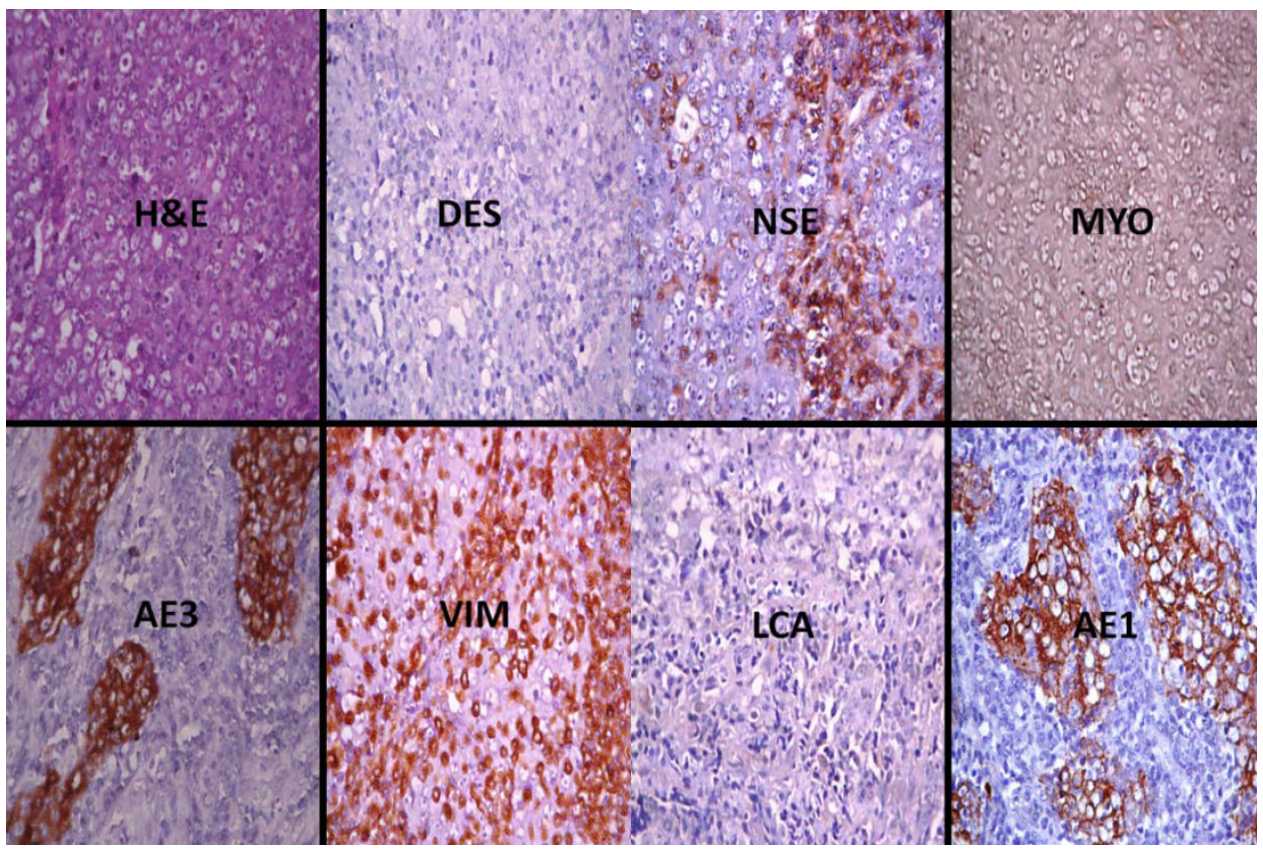

Figure 2 Photomicrographs showing the Immunohistochemical profile of a neuroendocrine carcinoma. The haematoxylin and eosin (H\&E) section shows highly pleomorphic cells with nuclei vessiculation and prominent nucleoli disposed in islands. Moderate immunopositivity of neurone specific enolase (NSE) for epithelioid-like cells is noted. Epithelial markers (AE1 and AE3) are strongly positive and there is a nonspecific staining pattern of epithelial cells and lymphocytes with vimentin (VIM). The tumour is negative for desmin (DES) and leukocyte common antigen (LCA). All immunohistochemical sections were counterstained with hematoxylin. (X400)

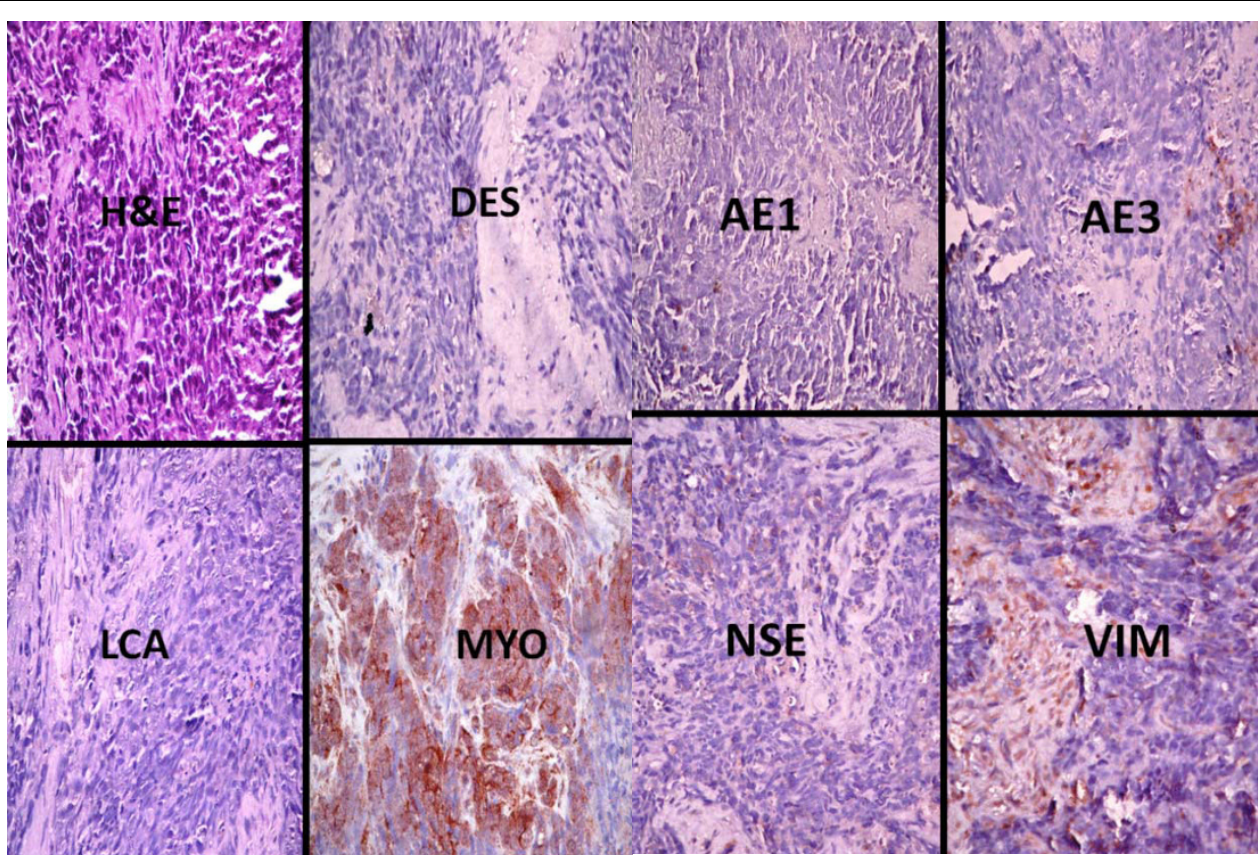

Figure 3 Photomicrographs showing the immunohistochemical profile of a rhabdomyosarcoma including the haematoxylin and eosin (H\&E) slide which displays sheets of pleomorphic and polymorphic cells which have hyperchromatic nuclei. There is strong immunopositivity for myogenin (MYO), moderate positivity for vimentin (VIM) and equivocal staining with desmin (DES) and neurone specific enolase (NSE). There is also negativity for epithelial markers (AE1 and AE3) and leukocyte common antigen (LCA). All immunohistochemical sections were counterstained with hematoxylin. (X400) 


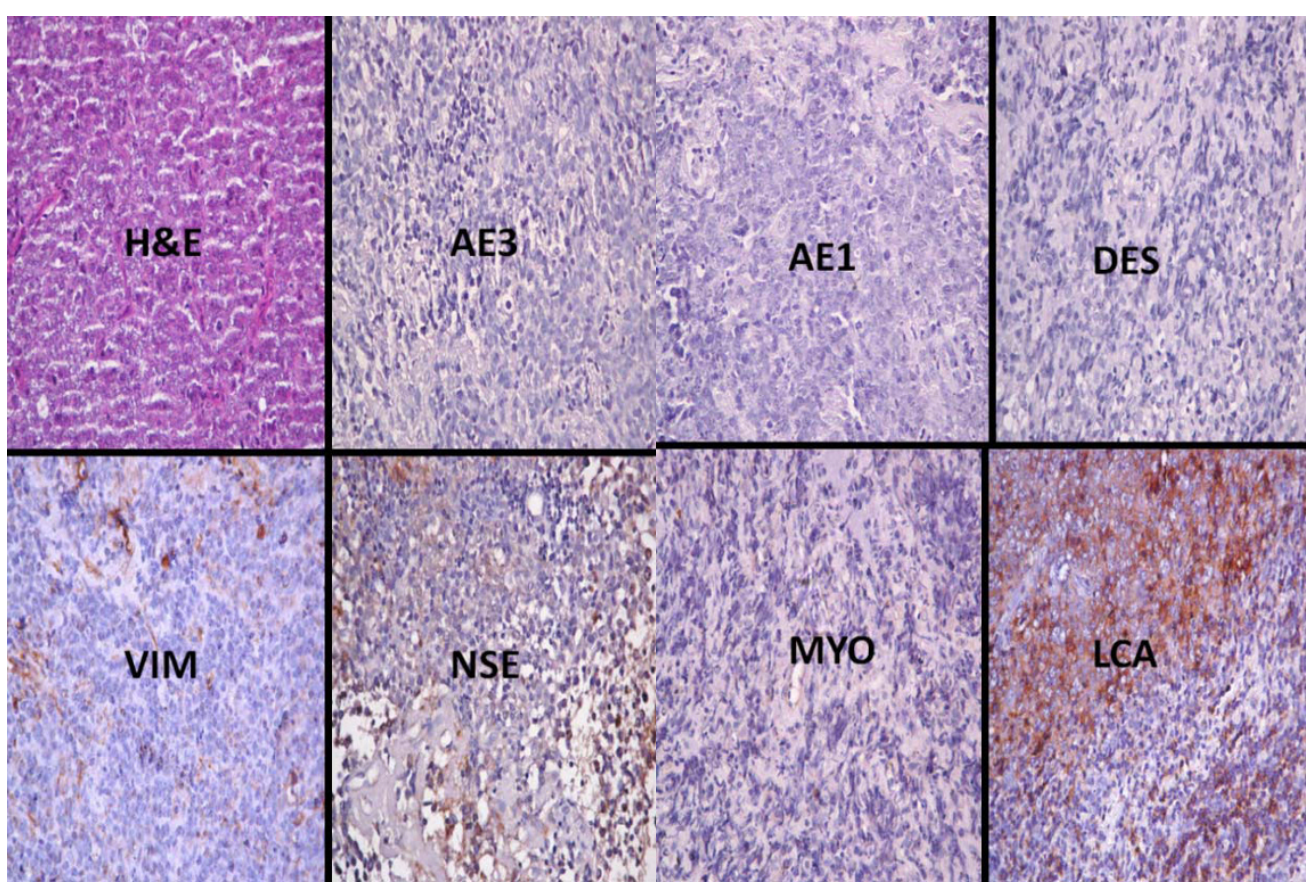

Figure 4 Photomicrographs show the immunohistochemical profile of a non Hodgkin's lymphoma including the haematoxylin and eosin (H\&E) slide that shows a highly cellular lesion with minimal supporting loose connective tissue stroma. There is strong

immunopositivity of the malignant lymphoid cells for leukocyte common antigen (LCA). All of the other immunohistochemical markers (AE1, AE3, myogenin (MYO), vimentin (VIM), desmin (DES), and neuron specific enolase (NSE) are negative. All immunohistochemical sections were counterstained with hematoxylin. (X400).

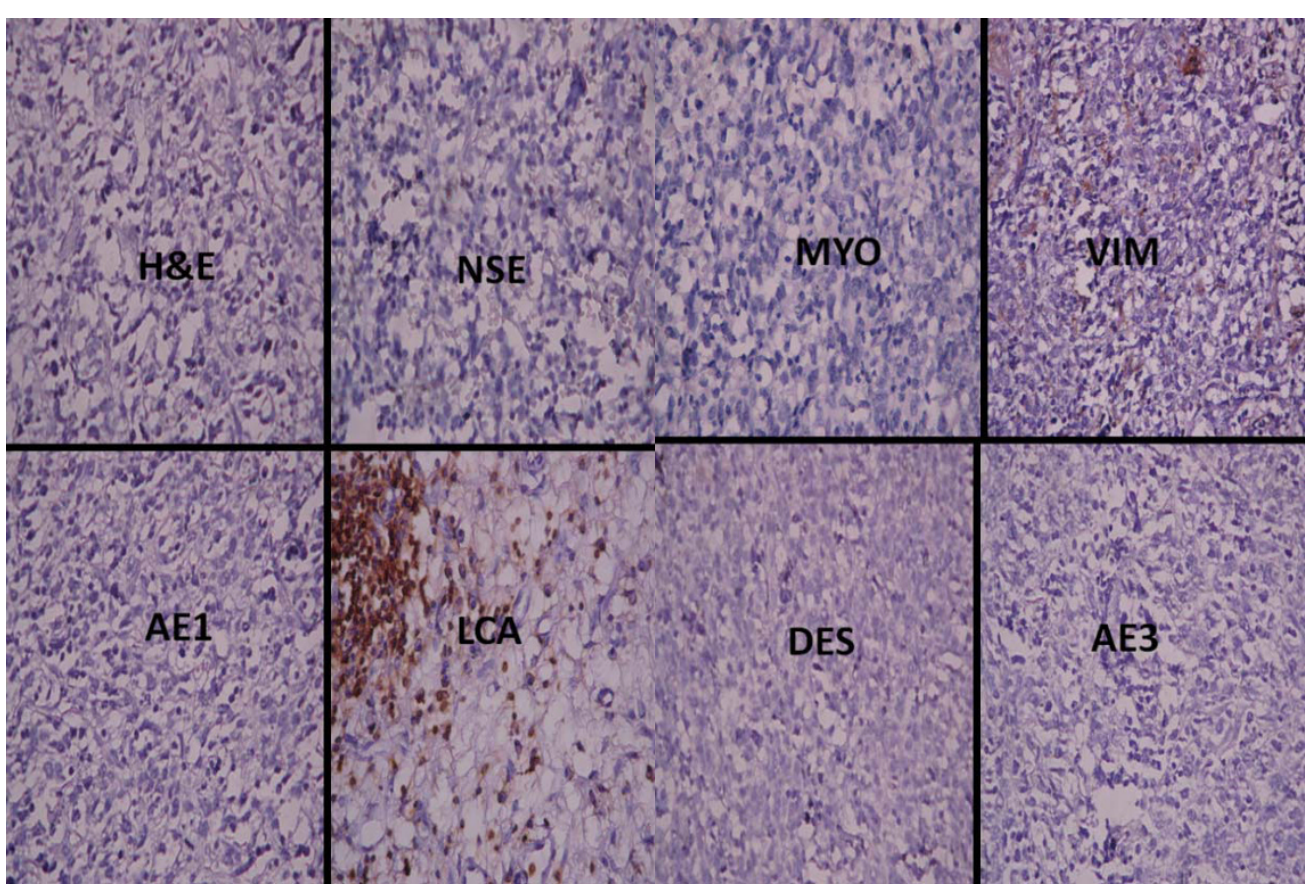

Figure 5 Photomicrographs are showing the immunohistochemical profile of a lymphoma including the haematoxylin and eosin (H\&E) slide which shows some small blue round and spindle cells with indistinct cytoplasm. There is immunopositivity for leukocyte common antigen (LCA). All of the other immunohistochemical markers are negative. All immunohistochemical sections were counterstained with hematoxylin. (X400). 


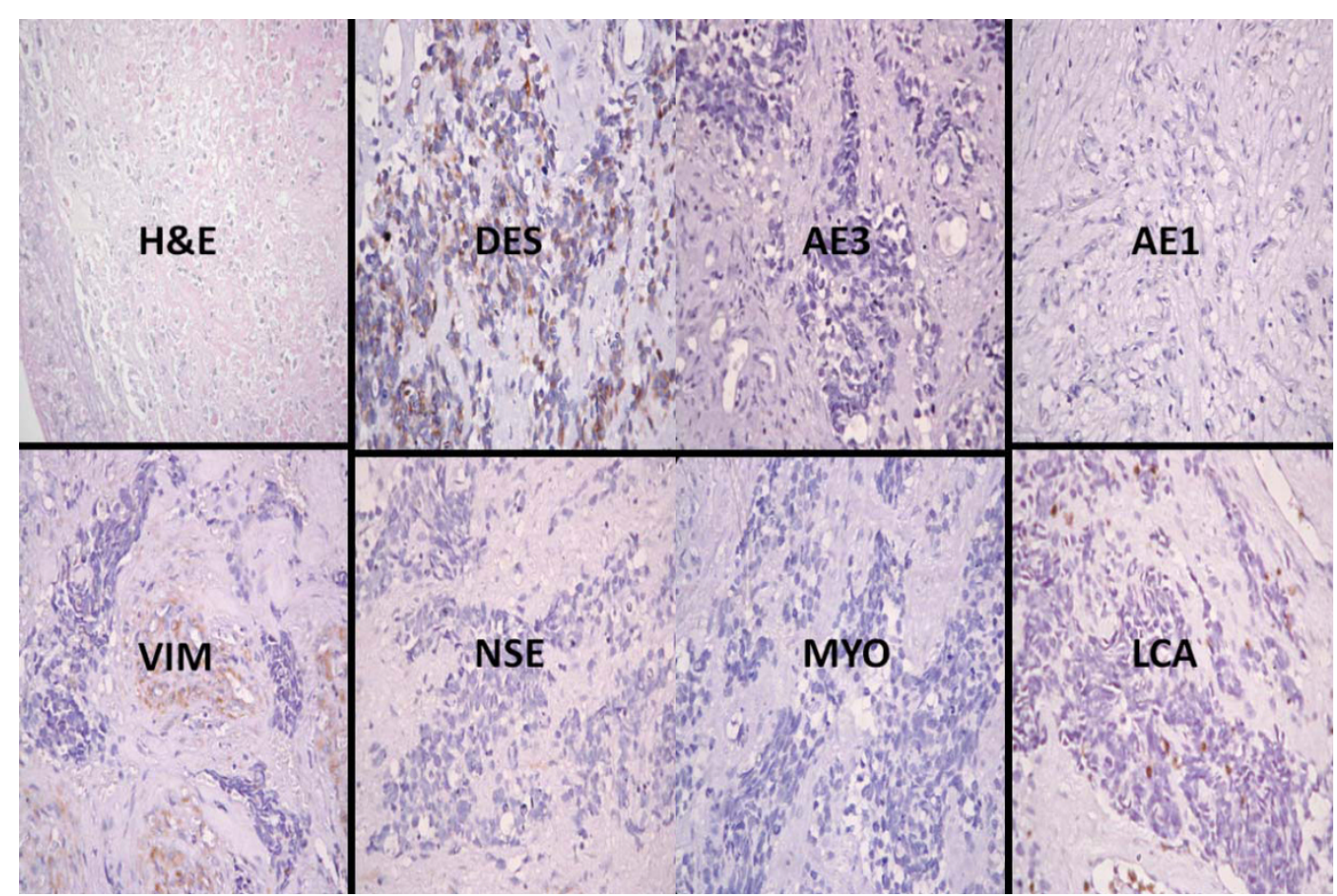

Figure 6 Photomicrographs show the immunohistochemical profile of a leiomyosarcoma including the haematoxylin and eosin (H\&E) slide. The islands of spindle cells with wavy nuclei show strong immunopositivity for desmin (DES). All of the other markers are negative, with fibrous connective tissue staining for vimentin (VIM). All immunohistochemical sections were counterstained with hematoxylin. (X400).

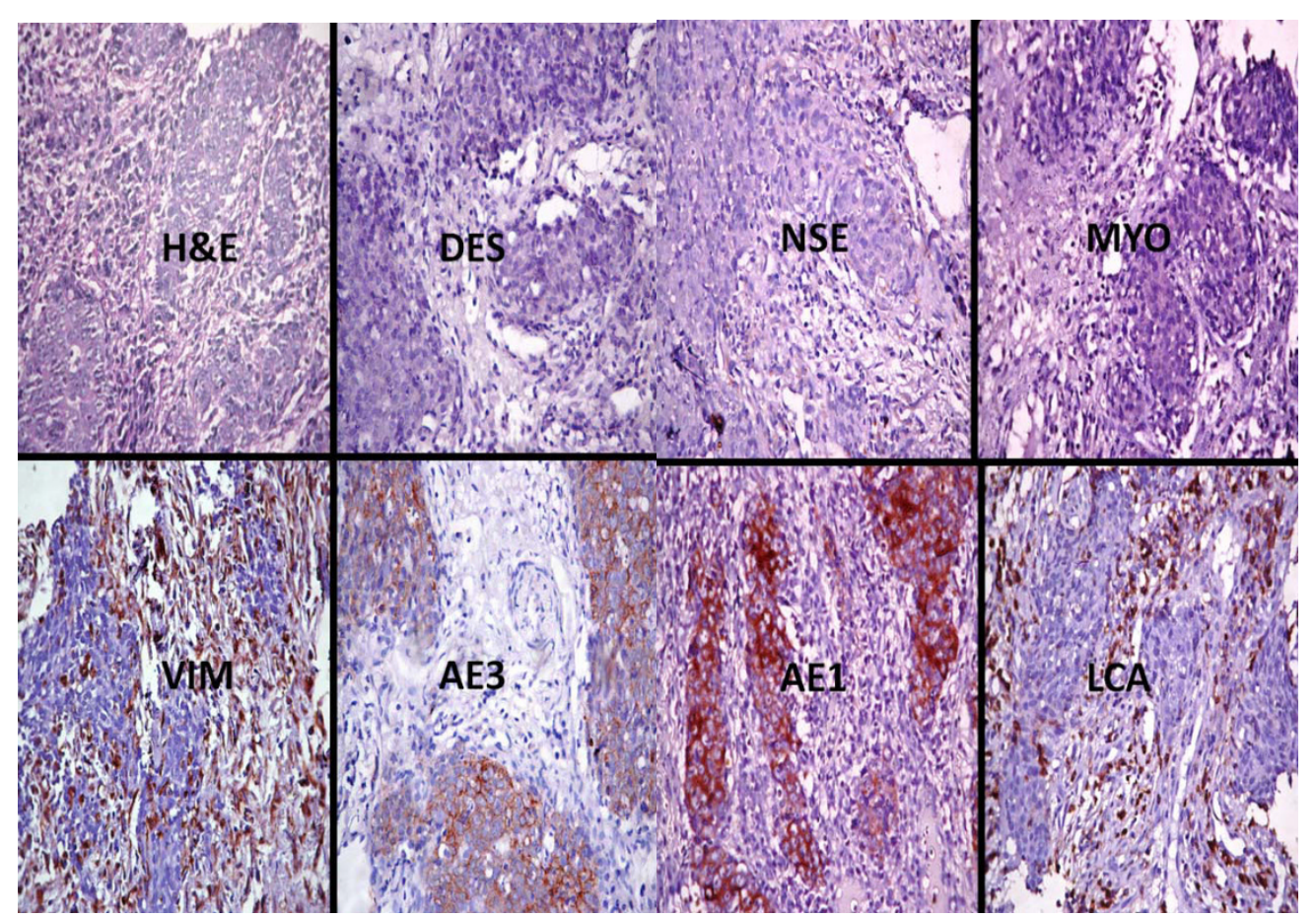

Figure 7 Photomicrographs show the immunohistochemical profile of a squamous cell carcinoma including the haematoxylin and eosin (H\&E) slide which show a follicular arrangement of small round hyperchromatic cells. There is strong immunopositivity for

cytokeratin AE1 and moderate positivity for AE3. All of the other immunohistochemical markers are negative. All immunohistochemical sections were counterstained with hematoxylin. (X400). 
Table 3 Sensitivity and specificity of histology

\begin{tabular}{lllll}
\hline & Carcinomas & Sarcomas & Lymphomas & Neuroendocrine carcinomas \\
\hline Sensitivity & 0.971 & 0.545 & 0.285 & 0.142 \\
Specificity & 0.477 & 0.947 & 0.984 & 0.986 \\
PPV & 0.596 & 0.800 & 0.800 & 0.500 \\
NPV & 0.954 & 0.843 & 0.864 & 0.922 \\
Accuracy & 0.696 & 0.835 & 0.860 & 0.911 \\
Kappa statistics value & 0.42 & 0.54 & 0.42 & 0.18 \\
\hline
\end{tabular}

PPV - positive predictive value, NPV - negative predictive value.

the choice of panel of antibodies based on cellular morphology to reduce 'wastage' of antibodies.

\section{Conclusion}

In this study, test of sensitivity and specificity show that histology is able to determine the presence or absence of a sarcoma or lymphoma to a good extent but confirmation of a carcinoma or neuroendocrine lesion is poor, although it is able to exclude them fairly accurately. This study has also shown that the use of antibodies in immunohistochemistry greatly assist in the identification of tumors which cannot be accurately identified using routine histopathological procedures.

More detailed studies for specific pathological conditions need to be carried out in order to maximize immunohistochemistry as an ancillary diagnostic tool and expand its versatility in Nigeria and the West African sub-region.

Conflict of interest statement

The authors declare that they have no competing interests.

\section{Authors' contributions}

$\mathrm{AO}, \mathrm{AO}$ and $\mathrm{JO}$ conceived of the study, and participated in its design and coordination and helped to draft the manuscript. EEU, BF and B participated in the design of the study, drafting of the manuscript and performed the statistical analysis. $\mathrm{AO}, \mathrm{AO}$ and EEU participated in the revision and reporting of the cases. All authors read and approved the final manuscript.

\section{Author details}

${ }^{1}$ Department of Oral Pathology, University College Hospital, Ibadan, Oyo

State, Nigeria. ${ }^{2}$ Department of Pathology, University College Hospital, Ibadan, Oyo State, Nigeria.

Received: 9 September 2010 Accepted: 3 November 2010 Published: 3 November 2010

\section{References}

1. Taylor CR: An exaltation of experts: concerted efforts in the standardization of immunohistochemistry. Appl Immunohistochem 1993, 1:232-243.

2. Ramaekers F: In Current perspectives on molecular and cellular oncology. Edited by: Spandidos D. JAI Press: London; 1992:285-318.

3. Gatter KC, Alcock C, Heryet A, Mason DY: Clinical importance of analyzing malignant tumors of uncertain origin with immunohistological techniques. Lancet 1985, 1(8441):1302-5.

4. Bahrami A, Truong LD, Ro JY: Undifferentiated Tumor: True Identity by Immunohistochemistry. Arch Pathol Lab Med 2008, 132(3):326-348.

5. Michaels L: Neuroectodermal tumors. In Ear, Nose and Throat Histopathology. Edited by: Michaels L. Berlin: Springer-Verlag; 1987:89.
6. Triche TJ: Diagnosis of small round cell tumors of childhood. Bull Cancer 1988, 75(3):297-310.

7. IHC World. [http://www.ihcworld.com/ihc_scoring.htm], Accessed on the 7/ 7/09.

8. Kirkland BR, Sterne JA: Essential of Medical Statistics., 2434

9. Bianchini WA, Altemani AM, Paschoal JR: Undifferentiated head and neck tumors: the contribution of immunohistochemical technique to differential diagnosis. Sao Paulo Med J 2003, 121:6.

10. Gatter KC, Alcock C, Heryet A, Pulford KA, Heyderman E, TaylorPapadimitriou J, Stein H, Mason DY: The differential diagnosis of routinely processed anaplastic tumors using monoclonal antibodies. Am J Clin Pathol 1984, 82(1):33-43.

11. Harrison LB, Sessions RB, Hong WK: Head and Neck Cancer. Google Books page 459 . Accessed on 28/July/2009.

12. Dar AU, Hird PM, Wagner BE, Underwood JC: Relative usefulness of electron microscopy and immunocytochemistry in tumor diagnosis: 10 years of retrospective analysis. J Clin Pathol 1992, 45(8):693-696

13. Dolan $M$, Snover D: Comparison of immunohistochemical and fluorescence in situ hybridization assessment of HER-2 status in routine practice. Am J Clin Pathol 2005, 123(5):766-770

doi:10.1186/1758-3284-2-33

Cite this article as: Adisa et al:: Immunohistochemical analysis of undifferentiated and poorly-differentiated head and neck malignancies at a tertiary hospital in Nigeria. Head \& Neck Oncology 2010 2:33.

\section{Submit your next manuscript to BioMed Central and take full advantage of:}

- Convenient online submission

- Thorough peer review

- No space constraints or color figure charges

- Immediate publication on acceptance

- Inclusion in PubMed, CAS, Scopus and Google Scholar

- Research which is freely available for redistribution 